\title{
Lait maternel ou lait de vache? \\ La production du corps de l'enfant par deux liquides nourriciers chez les Peuls du Ferlo (Sénégal)
}

\author{
Dorothée GUILHEM \\ Anthropologie sociale et culturelle: « Environnement, Santé, Sociétés »- UMI 3189, \\ Aix Marseille Université, Faculté secteur Nord, \\ 51, Bd Pierre Dramard, F-13916 Marseille Cedex 20 (France) \\ dorotheeguilhem@yahoo.fr \\ Gilles BOËTSCH \\ Anthropologie biologique: «Environnement, Santé, Sociétés »- UMI 3189, \\ Aix Marseille Université, Faculté secteur Nord, \\ 51, Bd Pierre Dramard, F-13916 Marseille Cedex 20 (France) \\ gilles.boetsch@gmail.com
}

Publié le 30 juin 2017

MOTS CLÉS

Lait maternel,

lait de vache,

enfance,

corps,
Peul,

Sénégal.

KEY WORDS

Breastmilk,

cow's milk,

childhood

body,

Fulani,
Guilhem D. \& Boëtsch G. - Lait maternel ou lait de vache? La production du corps de l'enfant par deux liquides nourriciers chez les Peuls du Ferlo (Sénégal), in Arena F., Foehr-Janssens Y., Papaikonomou I. \& Prescendi F. (éds), Allaitement entre humains et animaux: représentations et pratiques de l'Antiquité à aujourd'hui. Anthropozoologica 52 (1): 91-101. https://doi.org/10.5252/az2017n1a8

\section{RÉSUMÉ}

Dans la région du Ferlo au Sénégal, les femmes peules allaitantes introduisent précocement le lait animal dans le régime alimentaire des nourrissons. Ce mode de nourrissage des enfants en bas âge permet d'émettre une réflexion sur les relations de complémentarité, d'homologie ou de remplacement existantes entre les laits humain et animal, en fonction du statut et des rapports de proximité que ces pasteurs instaurent avec leurs bovins.

\section{ABSTRACT}

Breastmilk or cow's milk? Production of the child's body by two liquid foster among the Fulani of Ferlo (Senegal).

In the Ferlo region of Senegal, lactating women Fulani early introduce animal milk in the diet of infants. This method of feeding the infants used to introduce a reflection on the relations of complementarity, homology or replacing existing between human and animal milk, according to the special relationship that these pastors establish with their cattle. 


\section{INTRODUCTION}

Dans le corpus de littérature anthropologique sur les éleveurs et agro-pasteurs Peuls vivant en Afrique de l'Ouest ${ }^{1}$, la thématique de l'allaitement humain et animal n'a guère attiré l'attention des chercheurs. Si les nourritures enfantines ont suscité peu d'intérêt, les représentations et pratiques sociales relatives au lait animal ont constitué quant à elles un domaine de recherche privilégié. Elles s'inscrivent depuis les années 1960 dans un questionnement sur le pastoralisme, la répartition des activités techniques entre les sexes et les organisations socio-économiques qui en découlent (Dupire 1962a, b; Benoit 1982; Bonfiglioli 1988). Elles ont été reliées dans les contextes contemporains aux stratégies d'élevage et aux économies laitières localement adoptées depuis les crises écologiques et climatiques de 1972-1974 et de 1983-1985.

Les rapports entre les Peuls et leurs bovins, marqués par un fort attachement, ont également fourni un cadre d'analyse de leurs perceptions des ordres naturel, social et cosmologique. Les usages rituels du bétail et des produits laitiers ont permis d'appréhender la mise en correspondance de ces ordres dans la vie sociale (Dupire 1962b, 1970). Ils ont rendu compte de la participation du lait animal à des élaborations identitaires plurielles (Botte et al. 1999; Diallo \& Schlee 2000). Malgré la valeur heuristique de ces approches, les représentations et les utilisations du lait animal doivent être repensées en fonction du statut de l'enfant. La période de l'enfance éclaire sous un angle original le symbolisme alimentaire et social des laitages en fonction du processus de formation de cet être en devenir qu'est le nourrisson.

Dans les sciences humaines et sociales, la consommation de lait animal par les jeunes enfants est souvent envisagée en termes de complément ou de substitution au lait maternel. Cette interprétation prête à discussion car elle érige la pratique de l'allaitement et le lait maternel en norme de référence à partir de laquelle les autres nourritures enfantines sont questionnées. Or, la littérature anthropologique a déconstruit ce postulat en montrant que certaines sociétés n’assimilent pas le lait maternel à un aliment valorisé ou intrinsèquement adapté aux besoins de l'enfant (Malinowski 1930; Scheper-Hughes 1985; Fajans 1997). Elle a également démontré que des nourritures peuvent être investies d'un statut semblable à cette substance, voire d'une même capacité à produire des liens de parenté (Carsten 1999). La compréhension des rôles impartis aux laits humain et animal dans la petite enfance requiert ainsi de s'intéresser à leurs caractérisations respectives et cela, en fonction des spécifiques ontologiques propres aux groupes humains.

1. Les Peuls ou Fulbe (sing. pullo) se répartissent dans une quinzaine de pays de la zone subsaharienne de la Mauritanie et du Sénégal jusqu’au Tchad, Soudan et République Centrafricaine. Ils ont développé au cours de l'histoire différents degrés de mobilité (nomades, semi-nomades ou sédentaires), modes de subsistance (élevage ou agropastoralisme) et diverses exploitations des écosystèmes (chasse, cueillette, culture).
Dans la région du Ferlo au Sénégal, les femmes résidant dans les campements de Widou-Thiengoly allaitent leurs enfants mais introduisent précocement le lait animal dans leur régime alimentaire. Ce mode de nourrissage permet d'étudier la différente participation du lait humain et animal dans la production physiologique et sociale du corps de l'enfant. À partir de matériaux d'enquêtes anthropobiologiques menées auprès de femmes ayant toutes un enfant âgé de moins de six mois, nous proposons d'introduire les prémisses d'une réflexion ethnologique sur les relations de complémentarité, d'homologie ou de remplacement existantes entre ces deux liquides nourriciers.

\section{DU STATUT D'UN ANIMAL À UN MARQUEUR ALIMENTAIRE}

Dans la région du Ferlo, les Peuls se consacrent à l'élevage de bovins et de petits ruminants. Ils ont développé au cours des dernières décennies plusieurs degrés de mobilité en devenant éleveurs nomades ou pasteurs sédentaires (Barral et al. 1983; Pouillon 1990; Santoir 1994; Ancey et al. 2009). À l'élevage s'ajoute une pratique ancienne de cultures permettant d'assurer une meilleure subsistance et autonomie des groupes domestiques. Différentes combinaisons agro-pastorales ont été adoptées, selon que les pratiques agricoles soient équivalentes ou subordonnées aux impératifs d'élevage comme c'est le cas à Widou-Thiengoly.

Si les reconfigurations pastorales actuelles ont transformé durablement les modes de vie, elles ne reformulent guère le système de valeurs associé aux bovidés. Symboles de richesse et sources de prestige pour les hommes, les bovins constituent un capital social, symbolique et économique, que les pasteurs cherchent à préserver, à maximiser et à transmettre à leur descendance. Ils fournissent les ressources à la base de leur alimentation et de l'économie féminine, les produits laitiers. Les bovins sont peu présents dans les circuits d'échanges monétaires, mais ils circulent à l'intérieur de la société sous formes de prêts, de prestations matrimoniales et de droits d'héritage. L'économie laitière étant privilégiée, ils sont rarement abattus pour leur viande, excepté lors de grands événements tels que les mariages.

L'élevage des bovins s'inscrit pour les Peuls dans une logique de conciliation et de coopération des animaux (Boutrais 2002; Baroin \& Boutrais 2009). Ils octroient aux races bovines différentes compétences et qualités caractérielles (Dupire 1962a). Ils valorisent les vaches détenant un caractère sauvage mais qui, par apprivoisement et par une présence ancienne, se sont attachées aux éleveurs et savent se conformer à leurs attentes. Le gros bétail, par son statut économique et social, jouit d'une prééminence symbolique dans la hiérarchie animalière. Les analogies entre humains et animaux dans les chants apparaissent à cet égard significatives (Bovin 1999). Dans les chants de louanges, les pasteurs sont comparés en des termes élogieux aux taureaux ou aux vaches et non aux ovins ou aux caprins, animaux culturellement plus ambivalents. Les éleveurs peuvent aussi être 
représentés comme des propriétaires d'animaux dévalorisés, comme les ânes ou les chiens, lorsqu'il s'agit de tourner en dérision et de blâmer leur incompétence pastorale. Dans ce genre littéraire, la mise en ordre distinctive des animaux domestiques s'accompagne d'une énumération des valeurs et des comportements définitoires de l'image du bon ou mauvais berger.

L'élevage de petits ruminants au Ferlo s'est accru depuis les années 1960-1970. Pour certains Peuls, il présente une solution viable en raison du développement des surfaces agricoles et des difficultés d'accès aux pâtures. Pour d'autres, il contribue à une sécurisation de l'élevage de bovins, en permettant de faire face aux aléas climatiques et à la dégradation des ressources environnementales. À la différence des vaches, les ovins et caprins ont surtout une valeur d'investissement monétaire et d'échange, en soutenant les dépenses financières familiales. Jadis associé à la pauvreté, cet élevage procède aujourd'hui d'une gestion anticipée des risques et des formes de précarités sociales. Bien que leur statut symbolique soit peu réévalué, le petit cheptel représente une ressource alimentaire importante. Chèvres et moutons fournissent de la viande à l'occasion de visites et des fêtes religieuses.

Ces modes d'existence et d'économies familiales structurés autour de l'élevage expliquent la valorisation alimentaire du lait de vache (Bénéfice et al. 1984; Crenn et al. 2015). Les Peuls investissent les laits d'origine animale de différentes qualités gustatives, nutritionnelles et socio-symboliques. Ils accordent à Widou-Thiengoly leurs préférences alimentaires au lait de vache et de brebis, mais dédaignent le lait de chèvre et d'ânesse. La prise de laitages varie fortement selon les saisons. La saison des pluies, nommée "dunngu», est une période d'abondance caractérisée par une production laitière importante. Durant ces quelques mois, les produits laitiers constituent la base de l'alimentation quotidienne. Ils deviennent pour les femmes une source de revenus ou d'échanges contre des denrées alimentaires obtenues auprès de leurs voisins. Pendant la saison chaude «ceedu» du mois d'octobre à juin, le nombre de vaches laitières décroît, la majeure partie du lait est alors consommée par les familles. Lorsqu'elle est jugée insuffisante pour satisfaire leurs besoins alimentaires, les femmes ont recours au lait écrémé en poudre industriel.

Bien que dans les faits les Peuls consomment quotidiennement des céréales, ils se présentent comme des «buveurs de lait» et sont identifiés comme tels par les populations environnantes. Marqueur alimentaire, le lait de vache soutient l'élaboration et la production de stéréotypes mélioratifs permettant d'exprimer leur singularité culturelle. En dépit des réorganisations identitaires des éleveurs et des mutations alimentaires observables dans le Ferlo, ces discours stéréotypés maintiennent une distinction sociale voulue avec leurs voisins agriculteurs sédentaires. Dans ces dialectiques identitaires, le recours à la figure du pasteur transhumant, éleveur de bovins et buveur de lait, se présente comme un réinvestissement de la mémoire collective concernant l'origine des Peuls. Cette manipulation mémorielle revêt une importance particulière pour la compréhension du statut prêté au lait animal et à son rôle nourricier dans l'enfance.

\section{"ENTRE LE PEUL ET LA VACHE, C’EST LA PARENTÉ DE LAIT»}

Dans les mythes de cosmogénèse et d'anthropogenèse recueillis auprès de ces sociétés, la naissance des premiers Peuls coïncide souvent avec la domestication des vaches (Dupire 1962b; Seydou 1991). Selon les variantes, le troupeau primordial aurait une origine surnaturelle et aquatique ou il aurait été obtenu par la ruse en dépossédant d'autres populations de leurs vaches. Rapportée par Ba (2004), la légende d'Ilo Yeladi, ancêtre fondateur des différentes «familles " peules du Sénégal, présente l'originalité de relater de manière allégorique un allaitement interspécifique:

" "Ilo YelaDi" est un jaarga [grand propriétaire de bovins respecté de tous]. Il perd sa mère à la naissance. Son père est mort trois mois avant. On lui donne une nourrice, la nourrice meurt. On le nourrit au lait de chèvre, la chèvre meurt. Une femme de chez Diômel le prend en charge, sa calebasse remplie de lait se renverse par terre. Ilo était né porte-malheur (kiitaaDo) [...] Ilo est élevé dans la maison de Diômel qui est très riche en bétail et il est un "savant". Le fils de Diômel et lui sont très liés et ils ont le même âge. Diômel, lui, cependant, n'aime pas Ilo. Il n'apprécie surtout pas de le voir toujours présent au parc à bétail. Et Ilo, comme intuitivement persuadé de cela, l'évite. Arrive un jour où Diômel se trouva seul au parc avec son fils. Apparemment du moins. Il lui dit: "Tu vois la génisse une telle, là-bas? - Regarde." Diômel continue: "Le jour où elle sera pleine et qu' elle mettra bas, la première personne qui boira son lait à ce moment-là sera le plus grand parmi les plus grands de ce monde. M'as-tu entendu?" L'enfant dit: "Oui !". Et Diômel d'ajouter: "Mais où est donc Ilo, il n'est pas là, j’espère?" Le fils dit: "Non, il n'est pas là” Diômel insiste: “Tu es bien sûr, qu'il n'est pas là?" Le fils répond "Oui, je suis sûr”. Diômel ajoute: "Ceci est un secret, n'en rapporte rien à personne et surtout pas à Ilo". L'enfant dit: "Oui!"»(Ba 2004). Tandis que le père faisait ces recommandations, il ne remarqua pas la présence d'Ilo caché à proximité dans le parc à bétail. Malgré les directives paternelles, le fils délaisse la vache qui «se mit enfin à vêler. Il [Ilo] l'assista puis tira le lait bourru des mamelles chaudes et le but"( $\mathrm{Ba}$ 2004). À cette découverte et sous l'emprise de la colère, Diômel chasse de son foyer Ilo, la vache et son petit. Parti en brousse, «chaque jour que Dieu faisait, la vache revenait de pâture avec de nouvelles vaches. Il en fut ainsi de multiplication en multiplication, jusqu'à ce que Ilo devint le plus grand des plus grands parmi les Peuls» (Ba 2004).

Ce récit met en scène la figure de l'orphelin, être à la fois marginal et ambigü, dont les conditions d'existence malheureuses dans l'enfance augurent de sa destinée exceptionnelle. Dans les représentations sociales communes (Querre 2007), le nouveau-né orphelin durant cette période liminale peut être perçu comme un être potentiellement dangereux, en raison de son statut indéterminé, de sa proximité avec des entités surnaturelles et/ou de la concomitance de sa naissance avec des décès dans l'entourage familial. La légende se construit sur cette ambiguïté statutaire afin de restituer le parcours expérientiel de l'enfant. Ilo naît orphelin, privé de l'amour et de 
la protection parentale, puis il perd successivement ses deux nourrices, l'une humaine, l'autre animale, ce qui met sa vie en péril. Malgré son adoption dans une famille, son infortune perdure par la désaffection et la malveillance que lui voue Diômel. Cette inimitié est à l'origine de son bannissement et de son errance dans la brousse, avec pour seule compagnie la vache et son veau.

Suite à cette confrontation aux vicissitudes de la vie, le stratagème d'Ilo pour s'approprier le lait apparaît comme un acte à valeur initiatique, au cours duquel il perd son statut ambivalent d'orphelin pour acquérir celui d'ancêtre éponyme. Ce changement statutaire et identitaire est rendu possible par la manifestation de certaines dispositions intérieures. Les épreuves subies et surmontées par l'orphelin au fil des ans lui ont permis tout d'abord d'acquérir certaines vertus sociales. La conduite qu'il adopte lors de la ruse est à cet égard exemplaire, dans le sens où il a su dominer ses propres besoins physiques ou matériels pour veiller sur la vache et l'aider à mettre bas. Puis sa qualité d'éleveur se voit confirmée, puisqu'il noue des liens étroits avec le bovidé en ayant recours à des techniques pastorales d'accommodement. Son ascension produit un nouvel ordre social, à l'origine de la formation miraculeuse de son troupeau et de la création des "familles" peules du Sénégal. Elle légitime conjointement le savoir-faire pastoral "peul» au détriment de celui des Autres, à travers l'incompétence manifeste dont fait preuve le fils de Diômel. Le personnage d'Ilo incarne en cela un être porteur d'idéaux sociaux; ses dispositions morales - la patience, la résignation, le courage, la ténacité, l'indépendance, l'intelligence (associée à l'idée de ruse) - sont celles de l'archétype du pasteur valorisé par les éleveurs et qui donnent sens à l'élaboration idéologique de leur identité.

Cette légende expose une classification sociale des laits et leurs usages alimentaires. Privé du lait maternel à sa naissance, Ilo Yeladi survit grâce aux laits salvateurs de la chèvre et de la vache. Ces produits laitiers n'ont pas toutefois un statut analogue dans la légende. La mort prématurée du petit ruminant dénote la dévaluation sociale de cet animal et le peu d'attrait accordé à son lait. Elle soutient une mise en valeur socio-symbolique du lait de vache et du bovin "primordial». Ilo, qui n'a jamais goûté au lait maternel à sa naissance, s'inscrit véritablement dans le monde par l'ingestion de lait animal. Ce liquide nourricier est érigé en équivalent du lait maternel; tous deux sont sources de vie pour l'enfant et concourent à son intégration dans une communauté (ou à sa création). L'association entre le lait de vache et un principe vital est par ailleurs un motif récurrent dans la littérature orale des Peuls. Dans le texte initiatique "Koumen» des pasteurs sénégalo-gambiens, une goutte de lait animée par Guéno est à l'origine de la création du monde (Bâ Hampâte \& Dieterlen 1961), tandis que dans les contes camerounais ou maliens, le lait transformé en motte de beurre se métamorphose à son tour en un enfant (Eguchi 1994; Baumgardt 2000; Seydou 2005). Le lait animal apparaît ainsi comme une nourriture originelle et un substitut «culturel» des substances biologiques.
Le statut et les connotations symboliques de cet aliment invitent à considérer la consommation de lait d'Ilo comme un allaitement métaphorique interspécifique. Symbole d'élection, cet «allaitement» originel produit entre les pasteurs et les bovidés une parenté de lait. Il scelle l'union de l'homme et de l'animal en reflétant l'interdépendance matérielle, économique et socio-symbolique de leurs existences. Cet apparentement est évoqué à l'heure actuelle par les éleveurs pour caractériser le lien social établi avec leurs bovins: "entre le Peul et la vache, c'est la parenté de lait" ( hakkoundé pullo e naggue ko bandirâgal kosam) (Ba 2009: 1). On retrouve des qualifications analogues chez les Peuls Wodaabe du Niger « les bœufs sont nos parents de lait» (musiBBe) (Dupire 1970: 152) ou les Peuls Djelgôbé au Burkina Faso «la vache est le père du Peul» (nagge won baaba pullo) (Riesman 1992: 98).

L'«allaitement» d'Ilo n'est pas sans rappeler celui des enfants exposés nourris au pis de bêtes sauvages dans les mythologies antiques, les hagiographies médiévales ou la littérature folklorique (Roux 1967; Morel 1994; Parkes 2004; Dittmar et al. 2011). Ces allaitements interspécifiques ont en commun de souligner le caractère d'exception de ces enfants privés du sein maternel. Cependant, la traduction du mythe par Ba ne précise pas si Ilo s'abreuve directement au pis de l'animal, oriente le jet de lait vers sa bouche ou s'il utilise un récipient. Étant donné les normes régulant les prises alimentaires, il nous semble juste de penser que le héros ne tète pas le pis de l'animal. Cette technique consommatoire pour les Peuls rapproche l'être humain d'un état d'animalité ou d'ensauvagement, qui serait ici incompatible avec la figure de l'ancêtre fondateur. Elle traduit un manque de respect dû à l'animal et à son lait, qui s'illustre normalement dans les précautions prises lors de son recueil. Liquide précieux, il ne doit pas être versé à terre ni exposé aux éléments naturels, ce qui le rendrait impropre à la consommation, et encore moins refusé lorsqu'il est offert. La valeur symbolique de la vache se donne ainsi à voir à travers le traitement social de son lait.

\section{LES QUALITÉS CHANGEANTES DU LAIT MATERNEL}

Sur un plan narratif, un rapport d'équivalence entre le lait animal et humain est établi du fait qu'ils partagent un même statut fondateur de la vie. Cette relation doit être toutefois resituée dans un contexte social et confrontée aux propriétés que les femmes prêtent à la substance maternelle. Dans les conceptions locales du corps, le lait maternel est une substance dérivée conjointement du sperme, du sang et des apports nutritionnels des aliments. Il est jugé comme la meilleure nourriture pour le nouveau-né car le sang qui l'a fait croître in utero se transforme en lait à sa naissance et lui prodigue les mêmes bienfaits. Cette appréciation valorise une continuité biologique entre la grossesse et l'allaitement ainsi qu'une naturalisation du lien mère/enfant. Au-delà de ces représentations normatives, les caractérisations du lait apparaissent équivoques dès qu'elles sont reliées aux interprétations empiriques des femmes concernant la lactation. 
Selon de nombreuses femmes, le lait maternel se présente comme une substance intrinsèquement labile. Ses propriétés physiques, nutritionnelles et sanitaires se transforment tout au long de la période d'allaitement. Cette variabilité transparaît dans les distinctions faites entre le colostrum ( $k a n d i)$ et le «vrai» lait (kosam), puis entre un "bon» et un "mauvais» lait (moyya kosam et moyyi kosam). Comme pour d'autres sociétés ouest-africaines (Lestrange \& Passot-Guevara 1981; Dettwyler 1987; Bonnet 1988; Green etal. 1994; Duchesne 2000; Alfieri \& Taverne 2000), le colostrum est l'objet de représentations contrastées selon la prédominance accordée à certains registres de significations, physiologique, nutritionnel, sanitaire et religieux. Il est pensé par certaines femmes comme un liquide peu nutritif, mais suffisamment sain pour être ingéré par le nourrisson. Pour d'autres, il est impur et source de maux en raison de la présence de scories résiduelles à la grossesse. Si les Peuls du Séno, par analogie avec le monde animal, octroient au colostrum une grande valeur nutritive (Querre 2000), le "premier lait» pour les femmes de Widou-Thiengoly est davantage considéré comme une substance corporelle, plutôt que comme une nourriture à part entière.

Les qualifications de «bon» et «mauvais» lait confondent tant la qualité que la quantité de lait produite par le corps maternel. Un "bon» lait est blanc, consistant, tiède et "doux" au goût. Il est produit en quantité appropriée pour satisfaire l'appétit de l'enfant et il se modifie au cours de sa croissance pour s'adapter à ses besoins nutritionnels. Le lait maternel est dit "mauvais» lorsqu'il détient une teinte très claire ou blanchâtre-grisâtre, une consistance liquide comme de l'eau ou trop épaisse, ainsi qu'un goût insipide ou amer. Il est associé à une faible valeur nutritionnelle causant un amaigrissement de l'enfant, et peut même dans des cas précis, être jugé mortifère pour le nourrisson.

Ces altérations des qualités du lait ont différentes causes reconnues. Elles mettent en évidence le rôle important joué par le sang, substance principale autour de laquelle s'organisent les représentations de l'état de santé. Tout état maladif, en perturbant la circulation des substances et/ou le fonctionnement organique du corps, peut changer la valeur nutritive du lait et provoquer son tarissement partiel ou complet. La rupture de l'interdit de rapports sexuels ou une nouvelle grossesse au cours de la période d'allaitement modifient également ses qualités nutritionnelles et sanitaires. Lors des rapports sexuels, le sperme dans la matrice communique sa forte chaleur au sang, puis au lait dont il dénature le goût. Dans le cas d'une grossesse, la masse sanguine du corps est pensée insuffisante pour nourrir à la fois le fotus et l'enfant au sein. L'apport diminué en sang durant le processus de lactation rend la sécrétion laiteuse moins abondante, indigeste ou nocive. On retrouve ici la représentation répandue en Afrique, concernant l'incompatibilité entre l'acte d'alimenter un fotus et un enfant au sein, sous peine de porter préjudice à l'un des deux (Rabain 1979; Cosminsky 1985; Erny 1988; Fortier 2001; Mignot 2002; Walentowitz 2002; Kamudoni \& Gerd 2011).

Outre ces désordres physiologiques, la capacité à produire un «bon" lait est influencée par les conditions de vie et l'environnement social des femmes allaitantes. Les émotions fortes et négatives, telles que la souffrance causée par un décès, la colère suscitée par des conflits familiaux ou l'angoisse résultant de violences structurelles ou ordinaires, concourent à rendre le lait indigeste. Ce rapport causal, que l'on retrouve dans d'autres contextes culturels (Scheper-Hughes 1985; Farmer 1988; Fine 1994; Tapias 2006), induit le fait que pour remplir leur rôle nourricier, les femmes doivent être dans des conditions matérielles et sociales favorables.

Les perceptions féminines du lait sont empreintes d'ambivalence, à la fois positives et négatives, mais non réductibles à l'une ou à l'autre. Sa nature paradoxale est tributaire des modalités de la rencontre des substances dans le corps et de leur circulation entre les corps. Ces mises en contact des fluides expliquent l'existence de caractérisations différenciées du lait durant la lactation. Elles produisent tantôt une substance nourrissante et saine, tantôt un liquide empoisonné. Malgré ces aspects antinomiques, le continuum entre le sang et le lait font de lui la nourriture la plus adaptée pour l'enfant. De nature différente, le lait animal ne peut avoir la même richesse en nutriments. Il n'entretient pas dans cette perspective une relation d'équivalence avec le lait maternel, comme le suggère la légende, mais plutôt de complémentarité.

\section{LE RÔLE DU LAIT ANIMAL DANS LA PRODUCTION DU LAIT MATERNEL}

La fragilité de la fonction nourricière évoquée précédemment peut être régulée par des pratiques. Parmi celles-ci, le régime alimentaire des femmes allaitantes interagit de manière significative sur la lactation. Avant d'expliciter la contribution du lait animal dans la production du lait maternel, il nous faut brièvement décrire les habitudes alimentaires familiales.

Les trois repas journaliers se composent de préparations céréalières, principalement le mil et le riz, accompagnées de sauces. La composition des sauces peut allier des graines de légumineuses (arachides ou haricots niébé [vigna senensis]), des plantes alimentaires (oseille de Guinée, feuille de baobab, de casse fétide, de haricot dolique), des légumes (tomate, laitue, aubergine...), des tubercules, de la viande (bœuf ou mouton) ou du poisson frais, sec ou fumé. Du beurre de vache (nebam) est utilisé comme matière grasse dans la cuisson des sauces. Confectionné par les femmes, il se présente après le barattage sous la forme de petites mottes, ou sous celle d'une pâte semi-liquide lorsqu'il est cuit. D'autres préparations céréalières peuvent être consommées en plat principal, arrosées cette foisci de lait frais biraa'dam ou caillé keddam. Les couscous, mets très appréciés, se déclinent en diverses recettes selon les céréales choisies, leurs traitements culinaires et les procédés de cuisson usités. Ils acquièrent différentes saveurs selon l'adjonction des ingrédients, aigre, pimenté ou légèrement sucré.

Des bouillies semi-liquides à base de céréales et de lait frais, caillé, de petit lait ou de lait écrémé en poudre de fabrication industrielle, servent de collation au cours de la journée. Déjà décrites dans la littérature ethnologiques (Tourneux 2002; Sow 2005), elles sont confectionnées à partir de diverses farines ou semoule de mil, de petit mil, de riz ou de sorgho 
auxquelles on adjoint de l'eau et des condiments. Conservées un ou deux jours, elles peuvent se substituer aux mets mentionnés ci-dessus lorsque les femmes manquent de temps pour les activités culinaires. Outre ces collations, diverses boissons sont prises comme l'eau et le thé. Du lait frais est ingéré le matin avant le départ du troupeau pour les pâturages et le soir après le retour. Une autre boisson dénommée tufam, à base de lait caillé additionné d'eau et de sucre, est très prisée.

La consommation de ces mets à Widou-Thiengoly présente une grande variabilité en fonction du statut socioéconomique des familles, des lieux de résidence, des moyens d'approvisionnement et de conservation des denrées alimentaires. Elle est également tributaire de leurs disponibilités saisonnières. Les légumes sont peu présents durant la saison sèche, tout comme les produits laitiers dont l'utilisation alimentaire est réduite. Pendant la saison des pluies, les familles disposent au quotidien de grandes quantités de lait pour leur consommation domestique. Outre la prise de boissons et bouillies, elles peuvent manger à chaque repas une préparation céréalière avec un produit laitier. Les pratiques alimentaires adoptées durant la saison froide sont propices à l'obtention d'une lactation de bonne qualité. Ce régime alimentaire idéal pour les femmes témoigne du rôle important accordé aux laitages dans la production et la composition du lait.

La relation entre le lait maternel et le lait de vache transparaît dans les nourritures de l'accouchée. Le jour de l'accouchement, les parturientes ingèrent une bouillie tiède à base de lait de vache frais, de son de mil et de beurre. Puis elles consomment un plat de haricots niébé bouillis, mélangés à du beurre. Le don d'une bouillie contenant du lait de vache est attesté chez d'autres Peuls, ce qui témoigne de son caractère bénéfique (Dupire 1962b: 175; Querre 2000: 204). En effet, l'absorption de produits laitiers contribue à une épuration du sang en favorisant l'écoulement du " mauvais» sang et à déclencher la montée de lait. L'attribut assainissant du lait associé aux propriétés reconstituantes des céréales et des haricots redonnent au corps de la femme éprouvée sa vigueur initiale.

Durant la période de l'allaitement, la fabrication d'un lait maternel nourrissant et sécuritaire repose sur un imaginaire alimentaire relevant d'une pensée analogique. Comme dans d'autres sociétés ouest-africaines (Desclaux \& Taverne 2000; Mabilia 2005), les aliments de couleur blanchâtre sont investis de vertus galactogènes. Dans notre contexte, seul le lait de vache est considéré comme tel; la parenté étroite établie par les femmes entre les laits maternel et animal réside dans le fait qu'ils soient tous deux des éléments dispensateurs de vie et de formation corporelle, tant pour l'enfant que pour le petit animal. La prise régulière de laitages évite une dégradation des qualités du lait maternel ou son éventuel tarissement. Leur qualité de prolificité est telle que chez les Peuls Wodaabe du Niger, elle stimule la galactopoïèse chez une vieille grand-mère afin qu'elle puisse allaiter son petit enfant orphelin (Dupire 1957: 395).

Si le lait maternel est étroitement lié au lait des bovins, les autres nourritures participent davantage à la production du sang plutôt qu'à celle du lait. Dans les représentations physiologiques, la lactation est le produit de la chaîne de transforma- tion de la substance sanguine et de la nourriture absorbée par la mère. Chaque aliment ingéré concourt à la production du sang, dont les bienfaits nutritifs se répartissent différemment dans le corps durant la grossesse et l'allaitement. Quand la mère donne le sein, une partie des apports nutritionnels contribue au bon fonctionnement du corps maternel, une autre permet au sang de se muer en lait pour nourrir l'enfant. Des aliments comme les haricots sont réputés pour «donner du sang", c'est-à-dire que leur qualité tonique permet de produire à la fois du sang et du lait. Bien que des prescriptions alimentaires durant l'allaitement aient été relevées chez les Peuls (Dupire 1960: 66; Lestrange \& Passot-Guevara 1981; Tourneux 2007: 57), les femmes à Widou-Thiengoly préconisent des prises de nourritures régulières, abondantes et diversifiées. Cette recommandation s'explique au regard du régime de frugalité imposé par la saison sèche et des situations vécues de malnutrition causées par la pauvreté et l'insécurité alimentaire.

À la lumière de ce qui précède, l'une des principales caractéristiques du lait de vache est d'entretenir un rapport de contiguïté symbolique avec le lait maternel. Le lait animal ingéré par la mère se transforme en lait maternel, il régule ses qualités en fonction des éventuels désordres qu'elle peut subir et évite les formes d'hypogalactie. Proche de l'homologie, leur parenté étroite se fonde tant sur leurs propriétés physiques que sur leurs significations symboliques communes. Afin d'approfondir les correspondances entre ces deux nourritures, il nous faut préciser les modalités de la participation des produits laitiers dans la formation du corps de l'enfant.

\section{QUE COMMUNIQUENT LES PRODUITS LACTÉS AU CORPS DE L'ENFANT?}

Avant la première mise au sein, l'une des parentes de la lignée paternelle réalise sur le nouveau-né le rite musulman du « toxantal». Ce rite consiste à lui administrer dans la bouche trois gouttes de lait de chèvre mélangées à de l'eau, dans laquelle ont été préalablement dilués des versets coraniques écrits sur un morceau de papier. Il place l'enfant, être vulnérable, sous la protection d'Allah en l'affiliant à la communauté musulmane. L'utilisation du lait de chèvre obéit à une intentionnalité prophylactique et propitiatoire. Elle contribue tout d'abord à l'expulsion, à travers les selles, des impuretés utérines accumulées durant la période de gestation. Puis, elle influence favorablement la destinée future de l'enfant qui deviendra en grandissant aussi vif d'esprit, remuant et agile qu'une chèvre.

Excepté cette consommation rituelle, l'ingestion du lait de chèvre est dévaluée en raison de ses attributs olfactif et gustatif. Son odeur désagréable et son goût amer peuvent provoquer chez les enfants un dégoût pour les nourritures autres que le lait maternel. Il dessert dans cette perspective une logique de diversification alimentaire devant conduire au sevrage. Sa dévalorisation procède également d'une gestion de risques sanitaires. Le lait de chèvre est associé à la transmission chez l'enfant de pathologies comme la tuberculose, l'eczéma ou la diarrhée. Ces représentations alimentaires, oscillant entre le 
comestible et le toxique, soutiennent cette fois-ci une image négative de l'animal. Les chèvres apparaissent comme des animaux à l'hygiène douteuse et prêtant peu d'attention à la qualité des pâturages, ce qui rejaillit sur la nature suspecte de leur lait.

Ces jugements dépréciatifs formulés à l'égard du lait de chèvre procèdent d'une mise en valeur du lait de vache. Il est offert en complément du lait maternel entre le premier et le sixième mois (Sougou Gueye \& Boetsch 2015). Les bouillies à base de mil ou de riz et de lait sont données vers le neuvième mois de manière ponctuelle puis régulière. Un ordre semblable d'introduction de ces nourritures lactées a été observé chez d'autres Peuls, mais à des âges sensiblement différents (Dupire 1960: 65; Tourneux \& Yaya 1998: 299; Regis 2003: 97; Querre 2007). Cette diversification alimentaire est motivée par l'idée que le lait maternel devient insuffisant au fil des mois pour satisfaire les besoins nutritionnels nécessaires à la croissance du bébé. Or le lait de vache dans le symbolisme alimentaire local figure parmi les aliments détenant une haute valeur nutritive. Sa teneur élevée en matières grasses assure une prise de poids progressive et déjoue les dangers d'une éventuelle malnutrition.

Outre ses enjeux liés à la croissance, la consommation du lait de vache par les jeunes enfants est au cœur des enjeux d'une éducation au goût. L'expérimentation du plaisir sensoriel s'avère à cette période de la vie cruciale pour la survie de l'enfant. Elle doit susciter une curiosité gustative et procurer une satisfaction sensorielle devant conduire l'enfant à vouloir s'alimenter de lui-même. Le lait de vache est reconnu pour créer chez l'être humain une appétence. L'arrêt de sa consommation durant une longue période est par ailleurs associé à l'apparition de divers symptômes: démangeaisons, troubles de la vue ou maux de ventre. Par leur goût légèrement sucré et leur texture liquide ou semi-liquide, les préparations lactées éveillent ainsi l'appétit et attisent la gourmandise des enfants. Elles constituent une nourriture «transitoire» appropriée avant l'adoption des habitudes alimentaires des adultes.

Le lait de vache se présente comme une panacée naturelle. Ses propriétés curatives épurent et vivifient au quotidien le sang, ce qui offre à l'organisme du nourrisson une protection sanitaire. Sa vertu fortifiante contribue également selon les femmes à «ouvrir l'estomac». Cette expression se réfere à la fragilité de l'estomac et des intestins des enfants en bas âge. Ces organes doivent se fortifier et se développer pour que l'organisme puisse assimiler correctement les propriétés de la nourriture. Cette attention portée aux réactions physiologiques de l'enfant apporte un éclaircissement sur l'évitement de la consommation de lait de chèvre. À un âge trop précoce, la vulnérabilité de ces organes rendrait cet aliment indigeste, c'est pourquoi il est réintroduit dans les pratiques alimentaires des enfants vers l'âge d'un an et demi.

Durant les premiers mois de sa vie, la malléabilité attribuée au corps enfantin suggère qu'il peut être façonné selon des normes morphologiques et esthétiques. Les sept jours suivant sa naissance, des massages effectués avec du beurre de vache tendent à consolider les os de son squelette comme à renforcer la souplesse et la résistance de ses articulations. À cette attention prêtée à la fonctionnalité du corps se joint un souci d'ordre esthétique. La réitération de ces gestes permet d'arrondir la boite crânienne, d'affiner les traits du visage et d'allonger les membres corporels. Lors de ces manipulations du corps, l'application journalière du beurre de vache sur l'épiderme concourt à le raffermir, à le rendre brillant et à le protéger des effets néfastes de l'environnement naturel. L'ingestion du lait de vache et des bouillies dès le plus jeune âge procède d'une intentionnalité analogue à celle des massages. Elle permet à l'enfant d'acquérir une solide constitution physique devant favoriser l'acquisition de certaines aptitudes corporelles telles que l'agilité, la souplesse et l'endurance. Elle modifie durablement la couleur de l'enveloppe charnelle, qui devient claire, «rouge» et éclatante.

Symbole de santé et gage de beauté, les produits laitiers influencent positivement le développement des facultés cognitives et physiques des enfants. Ils agissent sur son activité métabolique, sur sa prise de poids et sur sa croissance de manière distincte du lait maternel. Si l'enfant hérite de certains attributs physiologiques et physiques de ses parents transmis lors de l'allaitement, la prise de laitages parfait ces apports initiaux pour garantir son bien-être et sa survie.

\section{LES NOURRITURES LACTÉES DANS LA FABRICATION DU LIEN SOCIAL}

La symbolique et les pratiques liées aux laits maternel et animal produisent des affects et des rapports sociaux participant à une fabrication sociale de l'enfant. Malgré la prévalence de la consanguinité dans la parenté et de la patrilinéarité dans la filiation, la pratique de l'allaitement réintroduit le rôle de la femme dans l'élaboration identitaire de l'enfant. Durant l'allaitement, la mère communique au nouveau-né des dispositions caractérielles et des aptitudes physiques. Le proverbe peul «ce que la vache a mangé, c'est cela que tête la génisse» ("ko nagge nami dum nale muinata») souligne la participation différenciée des substances maternelles et paternelles dans la production de l'enfant (Gaden 1931: 53). Bien que le lait soit un dérivé des substances des deux parents, la contribution maternelle semble prévaloir dans la transmission de traits de ressemblance du fait qu'elle allaite. Ainsi lorsqu'une mère se trouve dans l'incapacité de donner le sein, son choix va se porter sur une nourrice qui lui est apparentée afin de ne pas brouiller la transmission de ces traits de ressemblance. Ce choix préférentiel souligne les dangers encourus dans l'incorporation d'une substance non maternelle. Le lait «étranger» pourrait être générateur d'une confusion des identités en modifiant les dispositions intérieures ou les marqueurs corporels hérités à la naissance. Il peut altérer l'essence même du petit enfant en lui transmettant par exemple le pouvoir sorcier. L'ingestion du lait de la nourrice fait apparaitre les enjeux liés à la reconnaissance du partage d'une identité commune.

Si l'allaitement concourt à la transmission de la femme allaitante à l'enfant de traits de caractère ou d'aptitudes, il en est de même avec le lait animal. La consommation de lait de vache exerce une incidence positive ou négative 
sur les dispositions caractérielles des enfants. Les femmes à Widou-Thiengoly lui octroient la capacité de rendre les jeunes enfants éveillés, intelligents et obéissants. Cependant, le lait provenant de vaches possédées par des génies ou détenant une robe avec certaines combinaisons de couleurs est perçu comme dangereux. Il leur communiquerait des défauts tels qu'un tempérament colérique ou indiscipliné (Ogawa 1994). Plus largement, le lait de vache sert de métaphores descriptives de la détention de vertus sociales. Les expressions langagières «être de bon lait» ("moyya kosam») ou "être doux de lait» ("wela kosam») dénotent la possession de qualités morales telles que la noblesse, la réserve ou la patience. Selon Ba (2009), elles sont usitées dans les louanges faites aux femmes ayant contribué à la fortune de leur mari et à la réussite sociale de leurs enfants. Transposées dans le contexte de l'enfance, ces métaphores montrent que l'absorption du lait et de ses propriétés symboliques par l'enfant participe à une intériorisation et une incorporation de normes, de valeurs et de pratiques propre à la société à laquelle il appartient. En ingérant dès le plus jeune âge du lait, l'enfant construit dès lors par l'alimentation son identité socio-culturelle.

Ces nourritures lactées apparaissent comme des vecteurs de communication et de positionnement social au sein d'un groupe de parenté. Par l'enfantement, l'allaitement puis par son rôle d'éducatrice, la mère instaure un lien émotionnel et filial avec son bébé. Le lait maternel symbolise l'indéfectible lien d'amour filial et de protection unissant le nourrisson à sa mère et à un groupe de parenté utérine. Cet attachement est dépeint dans les pratiques langagières par l'image allégorique de l'allaitement ou du lait. Lorsqu'un adulte rend visite à ses parents après une longue absence, on dit littéralement chez les Peuls JafunBe et Djelgôbé du Burkina Faso qu'il vient "téter sa mère" (Riesman 1992: 123; Barry 2002: 138). Au Sénégal, les dissensions des liens familiaux ou le refus de remplir ses devoirs envers ses parents sont signifiés par l'acte de "couper le lait" ou "le sein" («tabude’ endam») (Gaden 1931: 7). Si ces images allégoriques insistent sur l'établissement d'un lien filial par l'allaitement, la circulation et le partage du lait animal traduisent dans les actes les relations de parenté. Ils symbolisent le degré d'affection, le partage de devoirs et de solidarités mutuels entre des membres apparentés. Ils permettent de surmonter des rivalités, de restaurer une communication menacée ou interrompue par des crises familiales. Ils concourent au quotidien à un resserrement des liens sociaux afin de renforcer un sentiment d'appartenance et une cohésion sociale.

Comme en témoigne la littérature ethnologique (Altorki 1980; Pauwels 1994; Jeudy-Ballini 1998; Conte 2000; Vernier 2006), diverses pratiques sociales liées au lait maternel permettent de constituer une parenté élective non fondée sur des liens biologiques. Très peu étudiée chez les Peuls, la parenté de lait atteste du pouvoir créateur de liens sociaux de cette substance. L'allaitement instaure un lien de parenté entre deux individus allaités par la même femme, avec ses apparentés mais aussi avec les autres enfants à qui elle a pu donner le sein. Il produit une prohibition d'alliances matrimoniales, interdit variable selon la prise en considération des consanguins et des affins de la nourrice. Au Niger, certaines femmes des Peuls WoBaaBe contrôlent le champ potentiel des alliances pour leurs filles, en refusant d'allaiter certains enfants mâles (Dupire 1970: 149). En Mauritanie (Ciavolella 2010: 171), les Fulaa’be des centres urbains instaurent une parenté de lait avec des enfants de familles voisines, appartenant à la communauté Maure, afin de créer de nouveaux liens de protection et de solidarité. Ces choix électifs illustrent les enjeux et les logiques sociales de la circulation du lait maternel entre les individus.

Comme le lait maternel, le lait animal permet lui aussi d'étendre un réseau de relations sociales. Les événements marquant les différentes étapes de la vie privée (imposition du nom, circoncision, mariage, funérailles) comme les fêtes religieuses annuelles sont célébrés par un repas composé de viande provenant d'un animal sacrifié et de lait frais ou caillé. La distribution et la commensalité développées autour du lait en ces occasions concourent à une mise en forme des rapports sociaux. Dans une gestion de l'altérité, le don de «tufam» ou de lait est une règle fondamentale de l'hospitalité. Symbole de richesse, il est un indice révélateur de la valeur de celui qui l'offre et de celui qui le reçoit et le boit. Lors des fêtes et des rassemblements, la consommation et le partage de nourritures lactées tissent des liens de sociabilité entre des individus et des groupes non apparentés. Ils instituent ainsi des formes de rapprochements et de collaborations en développant des rapports de fraternité, de confiance et de loyauté.

\section{CONCLUSION}

Les Peuls de Widou-Thiengoly utilisent un même terme «kosam» pour désigner le lait maternel et le lait animal. Cette dénomination commune reflète la relation d'unicité du principe vital partagée par ces deux liquides nourriciers. L'étymologie de ces termes en pulaar (ou fulfulde) corrobore le partage de ce même registre de significations. Selon Querre (2000), la classe nominale «dam» est à l'origine de plusieurs termes chez les Peuls du Séno au Burkina Faso: le lait «kosam», l'eau "ndiyam», le beurre "nebam», le sang «yiziyan» et la santé, le bonheur, la paix «jam». Chez les Peuls de Mauritanie, le terme "kosam» se serait substitué au mot «endam» ou «dendam» désignant le sein, le lait, la parenté utérine et les relations matrilatérales (Ciavolella 2010: 169). Dans son étude sur la parenté chez les Peuls JafunBe du Burkina Faso, Barry précise que le terme «kosan» désigne à la fois le lait maternel et le lait animal trait, offert ou vendu. Il ajoute que la notion de «dendan» doit être entendue comme tout «liquide laiteux à l'origine de», soit la sève des plantes, le colostrum de l'animal, le jeune lait de la mère et par extension, les liens de parenté (Barry 2002: 138). Malgré les différences dialectales observables selon les contextes géographiques, le lait maternel, substance corporelle à l'origine de la vie, est relié à une nourriture elle aussi pourvoyeuse de vie, de bien-être et de santé pour tout être humain, le lait de vache. Ces deux liquides nourriciers sont érigés en sources de vie et en symboles de fécondité. 
Les laits humain et animal apparaissent doublement fondateurs de la vie et de la personne selon qu'ils soient envisagés en termes d'aliment ingéré ou de substance. D'un point de vue alimentaire, ils jouent tout d'eux un rôle indispensable dans la vie de chaque être humain. Le lait maternel est un liquide dérivé du sang maternel et des aliments absorbés par la mère. Cette nourriture naturellement produite par le corps humain apparaît parfaitement adaptée pour favoriser la croissance et le bien-être de l'enfant. Ses propriétés à répondre différemment à ces besoins nutritionnels au cours de l'avancée en âge du nourrisson renforcent une telle représentation. Le lait de vache quant à lui partage des qualités matérielles et nutritives analogues au lait maternel. Il est le premier aliment offert à l'enfant afin de diversifier son régime alimentaire. Dans le cas de figure où le lait maternel est pensé moins riche en nutriments, il représente un aliment de choix pour compléter l'alimentation du bébé. En tant que composante essentielle de l'alimentation des Peuls, il sera consommé durant la totalité du cycle de vie.

Sous l'angle d'une théorie locale du corps, ces deux liquides se transforment dans l'organisme pour produire des substances vitales. Dans le corps du petit enfant, le lait humain et animal sont des nutriments contribuant à la production et au renouvèlement du sang dans son organisme. Le sang n'apparaît pas simplement comme une substance avec laquelle est né un enfant, il est perpétuellement transformé par la nourriture absorbée par la mère et par l'enfant lui-même. Dans le corps maternel, le lait de vache permet de déclencher la montée de lait et de changer le colostrum en un lait nourricier et sécuritaire. Il permet une régulation volontaire de la qualité et de la quantité de lait maternel afin de pourvoir aux besoins de l'enfant. Avec le beurre, il est un composant essentiel des recettes thérapeutiques luttant contre les troubles de la lactation. En ce sens, même si une femme détient la capacité "innée " à produire du lait maternel, celui-ci se voit continuellement créé par les aliments consommés, notamment par le lait animal. Cette chaîne de transformation du lait animal en sang dans le corps du nourrisson, puis en sang et lait dans le corps maternel, incite à repenser son statut alimentaire et symbolique.

Pour cela, il nous faut préciser les rapports entre le lait maternel, le lait de vache et la semence masculine. Dans les représentations du corps, "l'eau de l'homme» se concentre dans les articulations, la moelle des os et de l'épine dorsale. Elle se forme à partir de la même chaîne de transformation de la nourriture en sang évoquée précédemment. La consommation du lait de vache agit sur la production du sang mais aussi sur celle du sperme. Cette relation transparaît dans une recette médicinale contre l'impuissance masculine. Les hommes absorbent une bouillie à base de lait de vache et de moelle d'os de moutons diluée afin de recouvrer une vigueur sexuelle et être féconds. Le lait animal collabore ainsi à l'émission soit du sperme, soit du lait selon le sexe de ceux qui les absorbent. Lors des rapports sexuels, la semence se mue en sang dans la matrice et se mélange au sang "féminin».
Les deux substances, par un phénomène de coction, se coagulent et se transforment en un amas de chairs fotales. Le sperme est pensé comme un principe actif puisqu'il déclenche la conception d'un enfant mais aussi le processus physiologique de la lactation. Il partage une origine commune avec le lait maternel et ils sont tous deux associés à l'idée de croissance et de maturation. En dépit de cette participation du liquide séminal, on ne peut apposer au lait maternel un caractère sexué qui serait exclusivement masculin. Il apparaît davantage comme une substance bisexuée en raison du rôle octroyé au sang maternel et à la nourriture absorbée par la femme allaitante. Ces deux fluides entretiennent par ailleurs au cours de la période d'allaitement une relation d'opposition, puisque au contact du sperme les qualités du lait maternel s'altèrent.

Au regard de ses rapports avec le sang, le sperme et le lait maternel, le lait de vache entretient une relation de contiguïté symbolique avec les substances corporelles impliquées dans la procréation. De par son statut symbolique et ses propriétés, il ne saurait être envisagé de manière européocentrique comme un aliment de complément et de substitution. Le continuum instauré entre le lait animal et humain invite à les considérer comme des "substances partagées» dans l'acceptation qu'en donne Carsten (1999). Bien que de natures différentes, ces nourritures lactées participent de manière distincte mais complémentaire à la fabrication biologique et sociale du nourrisson. Leur contribution respective s'entremêle en communiquant des traits psychiques et morphologiques, sièges de son individualité et marqueurs de son appartenance sociale. Elle confère autant d'importance à la transmission de caractères héréditaires lors de la génération et de l'allaitement, qu'à l'acquisition et à l'incorporation, lors des prises quotidiennes de lait animal, de valeurs symboliques et idéologiques fondatrices de l'identité socio-culturelle des Peuls. Les laits humain et animal façonnent non seulement le corps de l'enfant mais concourent aussi à la formation de sa personne. Cette capacité s'étend à une constitution de l'enfant, en tant qu'être social relié à d'autres personnes par des formes de sociabilités et d'affects. Elle tisse un lien filial privilégié avec les membres du groupe de parenté utérine mais aussi avec ceux de la parenté agnatique, grâce à un échange nourricier de lait animal, empreint d'intimité et de générosité. Elle modélise ses relations sociales actuelles et futures avec des familles selon des rapports électifs et des prohibitions matrimoniales générées par la parenté de lait, puis selon des liens d'affinité créés par la commensalité autour du lait animal. Si ces nourritures contribuent à une fabrique de l'enfant par des «substances partagées" au sein de la parenté, elles montrent également que le partage d'un même ethos alimentaire avec des personnes non apparentées joue aussi un rôle essentiel dans la production de son identité. Les représentations et les pratiques liées aux laits humain et animal appellent ainsi à questionner la relation entre le biologique et le social, en ne les opposant pas mais en interrogeant plutôt leur rapport de continuité. 


\section{RÉFÉRENCES}

Alfieri C. \& TAVerne B. 2000. - Ethnophysiologie, règles et précautions chez les Bobo Madare et les Mossi, in DesCLAUX A. \& TAVERne B. (éds), Allaitement et VIH en Afrique de l'Ouest. De l'anthropologie à la santé publique. http://horizon. documentation.ird.fr/exl-doc/pleins_textes/pleins_textes_7/b_ fdi_03_02/010024738.pdf dernière consultation: 17/03/2017.

ALTORKI S. 1980. - Milk-kinship in Arab society: an unexplored problem in the ethnography of marriage. Ethnology 19 (2): 233244. http://www.jstor.org/stable/3773273

Ancey V., ICKOwicz A., Corniaux C., Manoli C. \& Magnani S. 2009. - Stratégies pastorales de sécurisation chez les Peuls du Ferlo (Sénégal). Journal des africanistes 78 (1-2): 105-119.

BA S.-M. 2004. - Dis-moi ce que tu manges et je te dirai qui tu es. Le lait des Peuls, Kosam Foulbé. Anthropozoologica 39 (1): 27-41.

BA S.-M. 2009. - Le lait des Peuls. Entre les pasteurs peuls et leurs vaches: une "parenté de lait». http://www.lemangeur-ocha.com/ wp-content/uploads/2012/04/Le-lait-des-Peuls.pdf dernière consultation: $17 / 03 / 2017$.

BÂ Hampâte A. \& Dieterlen G. 1961. — Koumen: texte initiatique des pasteurs peul. Mouton \& Cie, Paris, 95 p.

Baroin C. \& Boutrais J. 2009. - Bétail et société en Afrique. Journal des Africanistes 78 (1-2): 9-52.

Barral H., Bénéfice E., Boudet G., Denis J. P., Wispelaere G. (DE), Diaite I., Diaw O. T., Dieye K., Doutre M. P., Meyer J. F., Noël J., Parent G., Piot J., Planchenault D., Santoir C., Valentin C., ValenZa J. \& VAssiliades G. 1983. — Système de production d'élevage au Sénégal dans la région du Ferlo: synthèse de fin d'études d'une équipe de recherches pluridisciplinaire. Orstorm, Paris, 172 p. http://www.documentation.ird.fr/hor/fdi:04222 dernière consultation: 17/03/2017.

BARRY A. 2002. - Le lien de sève et la maison paternelle: aux racines de l'identité des Peuls JafunBe du pays samo. L'Homme 163: 137-156. https://doi.org/10.4000/lhomme.175

BAUMGARDT U. 2000. - Une conteuse peule et son répertoire: Goggo Addi de Garoua, Cameroun. Karthala, Paris, 552 p.

BÉNÉFICE É., Chevassus-Agnès S. \& BARRAL H. 1984. - Nutritional situation and seasonal variations for pastoralist populations of the Sahel (Senegalese Ferlo). Ecology of Food and Nutrition 14: 229-247.

BenolT M. 1982. - Nature Peul du Yatenga, remarques sur le pastoralisme en pays mossi. Orstom, Paris, 176 p. (Coll. Travaux et document de l'Orstom; 143)

Bonfiglioli A. M. 1988. - DuDal, histoire de famille et histoire de troupeau chez un groupe de WodaaBe du Niger. Maison des Sciences de l'Homme, Paris, $307 \mathrm{p}$.

BONNET D. 1988. - Corps biologique, corps social, procréation et maladies de l'enfant en pays mossi. Orstorm, Paris, $138 \mathrm{p}$.

Botte R., Boutrais J. \& SCHMitz J. 1999. — Figures peules. Karthala, Paris, 540 p.

Boutrais J. 2002. - Patrimoine animal et territoire chez des sociétés peules, in Cormier Salem M. C., Juhé-Beaulaton D., Boutrais J. \& Roussel B. (éds), Patrimonialiser la nature tropicale: dynamiques locales, enjeux internationaux. IRD, Paris: 167-188.

Bovin M. 1999. — La belle vache, chants de louange aux animaux et aux êtres humains chez les WodaaBe du Niger, in BoUTRAIS J. \& BAROIN C. (éds), L'homme et l'animal dans le bassin du Tchad. IRD, Paris: 202-219.

CARSTEN J. 1999. - The substance of kinship and the heat of the hearth: feeding, personhood and relatedness among Malays in Pulau Langkawi. American ethnologist 22 (2): 223-241.

Ciavolella R. 2010. - Les Peuls et l'État en Mauritanie: une anthropologie des marges. Karthala, Paris, $432 \mathrm{p}$.

CONTE E. 2000. - Les lois du genre: approche comparative des systèmes de parenté arabe et touareg, in JAMARD J-L., TERRAY E. \& XANTHAKOU M. (éds), En substances. Textes pour Françoise Héritier. Fayard, Paris: 135-156.
COSMINSKY S. 1985. — Infant feeding practices in rural Kenya, in Hull V. \& Simpson M. (éds), Breastfeeding Child Health and Child Spacing: cross-cultural perspectives. Croom Helm, London, Dover: 35-54.

Crenn C., KA A. \& Leport J. 2015 - Entre brousse, ville et globalisation: les paysages alimentaires du Sénégal, in BOETSCH G., GuEYE L., JAFFRE Y. \& MACIA E. (dir), Santé et société en Afrique de l'Ouest. CNRS Éditions, Paris: 171-201.

DesclauX A. \& TAVERNE B. 2000. - Allaitement et VIH en Afrique de l'Ouest: de l'anthropologie à la santé publique. Karthala, Paris, 556 p.

DeTTWYLER K. A. 1987. - Breastfeeding and weaning in Mali: cultural context and hard data. Social Science \& Medecine 24 (8): 633-644. https://doi.org/10.1016/0277-9536(87)90306-6

Diallo Y. \& SCHLEE G. 2000. - L'ethnicité peule dans des contextes nouveaux. Karthala, Paris, 255 p.

DitTmar P.-O., Maillet C. \& Questiaux A. 2011. — La chèvre ou la femme: parentés de lait entre animaux et humains au Moyen Âge. Images Re-vues 9. http://imagesrevues.revues.org/1621 dernière consultation: 17/03/2017.

DuCHESNE V. 2000. — Grossesse et allaitement en milieu anyi, in Gruénais M.-C., JafFré Y. \& Olivier de SARdAN J.-P. (éds), Grossesse, contraception, avortement, accouchement en Afrique de l'Ouest (Atelier d'Abidjan, 4 et 7 novembre 1999). http://horizon.documentation.ird.fr/exl-doc/pleins_textes/divers14-11/010031071. pdf dernière consultation: 17/03/2017.

DuPIRE M. 1957. - Pharmacopée peule du Niger et du Cameroun. Bulletin de l'IFAN 19 (3-4): 380-415.

Dupire M. 1960. - Situation de la femme dans une société pastorale (Peul WoDaBe, Nomades du Niger), in PAulme D. (dir.), Femmes d'Afrique noire. École Pratique des Hautes Études; Mouton \& Cie, Paris: 51-92.

DuPIRE M. 1962a. — Des nomades et leur bétail. L'Homme 2 (1): 22-39.

Dupire M. $1962 \mathrm{~b}$. - Peuls nomades. Étude descriptive des WoDaabe du Sahel nigérien. Karthala, Paris, 336 p.

DuPIRE M. 1970. — Organisation sociale des Peuls. Plon, Paris, 617 p.

EGuCHI P. 1994. - Pastoralism in fulbe folktales. Cahiers d'Etudes africaines 34 (133-135): 461-472.

ERNY P. 1988. - Les premiers pas dans la vie d'un enfant d'Afrique noire. L'Harmattan, Paris, $360 \mathrm{p}$.

FajAnS J. 1997. - They Make Themselves: Work and Play Among the Baining of Papua New Guinea. University of Chicago Press, Chicago, $328 \mathrm{p}$.

FARMER P. 1988. — Bad blood, Spoiled milk: bodily fluids as moral barometers in rural Haiti. American ethnologist 15 (1): 62-83.

Fine A. 1994. — Le lait "contrarié», in GILLET P. (dir.), Mémoires lactées. Blanc, bu, biblique: le lait du monde. Autrement 143: 157170. (Coll. Mutations/Mangeurs) http://www.mangeur-ocha. $\mathrm{com} /$ fileadmin/contenusocha/09_lait_contrarie.pdf dernière consultation: 17/03/2017.

ForTiER C. 2001. - Le lait, le sperme, le dos, et le sang ? Représentations physiologiques de la filiation et de la parenté de lait en islam malékite et dans la société maure. Cahiers d'Études africaines (161): 97-138.

Gaden H. 1931. - Proverbes et maximes peuls et toucouleurs. Traduits, expliqués et annotés. Institut d'ethnologie, Paris, 368 p.

GreEn E., Jurg A. \& DJedje A. 1994. - The snake in the stomach: child diarrhea in central Mozambique. Medical Anthropology Quarterly 8 (1): 4-24. http://www.jstor.org/stable/648989

JEUDY-BALLINI M. 1998. — Naître par le sang, renaître par la nourriture, in Fine A. (éd.), Adoptions: Ethnologie des parentés choisies. Maison des sciences de l'homme, Paris: 19-44.

KAMUdONI P. \& GERD H.-O. 2011. — From traditional to optimal breastfeeding practices: selected cases from Central and Southern Africa, in LiamputTong P. (éd.), Infant Feeding Practices: A Cross-Cultural Perspective. Springer, Victoria: 231-246.

Lestrange M. T. de \& Passot-Guevara B. 1981. — De la grossesse au sevrage, chez les Bassari, les Peul et les Boïn de Salemata (Sénégal oriental). Journal des africanistes 51 (1-2): 23-41. 
MabiLia M. 2005. - Breast Feeding and Sexuality. Behaviour, Beliefs and Taboos Among the Gogo Mothers in Tanzania. Berghahn Books, United States, $176 \mathrm{p}$.

MALINOWSKI B. 1930. — La vie sexuelle des sauvages du nord-ouest de la Mélanésie. Payot, Paris, 405 p.

Mignot J.-M. 2002. — Le sevrage chez les Masa Bugudum de la région de Nouldayna (Cameroun). Journal des africanistes 72 (1): $19-30$.

Morel P. 1994. — Lait des faibles et lait des forts, in GILlET P. (dir.), Mémoires lactées. Blanc, bu, biblique: le lait du monde. Autrement 143: 74-86. (Coll. Mutations/Mangeurs). http://www.lemangeurocha.com/fileadmin/contenusocha/05_lait_faibles_lait_fort.pdf dernière consultation: 17/03/2017.

OGAWA R. 1994. - Gaabgol et kuumeen: cohésion sociale et disparités économiques. Cahiers d'études africaines 34 (133-135): 281-293.

PARKES P. 2004. - Fosterage, kinship, and legend: when milk was thicker than blood? Comparative Studies in Society and History 46 (3): 587-615. https://doi.org/10.1525/maq.1994.8.1.02a00020

PAUWELS S. 1994. - L'adoption: le travail rituel des relations de germanité dans une société d'Indonésie orientale, in KOUBI J. \& MASSARD-VINCENT J. (éds), Enfants et sociétés d'Asie du sud-est. L'harmattan, Paris: 65-81.

Pouillon F. 1990. - Sur la stagnation technique chez les pasteurs nomades. Cahier des sciences humaines 26 (1-2): 173-192.

QUeRRE M. 2000. — La voie du lait: le sens des pratiques autour de l'allaitement chez les Peuls du Séno, in DesClaux A. \& TAVERnE B. (dirs), Allaitement et VIH en Afrique de l'Ouest: de l'anthropologie à la santé publique. Karthala, Paris: 189-216.

Querre M. 2007. - Enjeux sociaux autour du corps de l'enfant: l'exemple des Peuls du Séno Burkinabé. Face à face 10. http:// faceaface.revues.org/95 dernière consultation: 17/03/2017.

Rabain J. 1979. - L'enfant du lignage: du sevrage à la classe d'âge chez les Wolof du Sénégal. Payot, Paris, 237 p.

Regis H. 2003. - Fulbe Voices, Mariage, Islam and Medecine in Northern Cameroon. Western case, Colorado, $204 \mathrm{p}$.

RIESMAN P. 1992. - First find your Child a good Mother. Rutgers University Press, New-York, 264 p.

Roux J.-P. 1967. - Le lait et le sein dans les traditions turques. L'Homme 7 (2): 48-63. http://www.jstor.org/stable/25129731
SANTOIR C. 1994. — Décadence et résistance du pastoralisme: les Peuls de la vallée du fleuve Sénégal. Cahiers d'Études africaines 34 (133-135): 231-263.

SCHEPER-HugHES N. 1985. - Culture, scarcity, and maternal thinking: maternal detachment and infant survival in a Brazilian shantytown. Ethos 13 (4): 291-317. http://www.jstor.org/stable/640147

SEYDOU C. 1991. - Bergers des mots, poésie peule du Mâssina. Classiques africains, Paris, $257 \mathrm{p}$

SeYdOU C. 2005. - Contes peuls du Mali. Karthala, Paris, 496 p.

Sougou M. N. Gueye A. \& Boetsch G. 2015. — Diversification alimentaire et impact sur la croissance chez les enfants peuls de 0 à 5 ans dans le Ferlo, in Boetsch G., Gueye L., Jafrré Y. \& Macia E. (dirs), Santé et société en Afrique de l'Ouest. CNRS Éditions, Paris: 203-220.

Sow S. A. 2005. - Le lait, patrimoine des Peuls pasteurs du Niger: pratiques alimentaires, représentations et usages non alimentaires chez les Gaawoo'be du Gourma, in Cormier SALEM M.-C., JuHÉBeaulaton D., Boutrais Jean \& Roussel B. (éds), Patrimoines naturels au Sud: territoires, identités et stratégies locales: 419-442. http://www.documentation.ird.fr/hor/fdi:010037553 dernière consultation: 17/03/2017.

TAPIAS M. 2006. - Emotions and the intergenerational embodiment of social suffering in rural Bolivia. Medical Anthropology Quarterly 20 (3): 399-415.

TOURnEUX H. \& YAYA D. 1998. - Dictionnaire peul de l'agriculture et de la nature (Cameroun). Karthala, Paris, 542 p.

Tourneux H. 2002. - Les préparations culinaires chez les Peuls du Diamare (Cameroun), in RAIMOND C., GARINE E. \& LANGLOIS O. (éds), Ressources vivrières et choix alimentaires dans le bassin $d u$ lac Tchad. IRD, Paris: 289-318.

Tourneux H. 2007. - Dictionnaire peul du corps et de la santé. Karthala, Paris, 614 p.

VERNIER B. 2006. - Du bon usage de la parenté construite avec des humeurs corporelles (sang et lait) et quelques autres moyens. European Journal of Turkish Studies 4. http://ejts.revues.org/623 dernière consultation: 17/03/2017.

WALENTOWITZ S. 2002. - Lait d'honneur et seins charitables. À propos des pratiques d'allaitement non maternel chez les Touaregs de l'Azawag, in Bonnet D., Le GrandSébille C. \& Morel M.-F. (éds), Allaitements en marge. L'Harmattan, Paris: 111-140. 